\title{
LA EVALUACIÓN PARA EL APRENDIZAJE EN LA EDUCACIÓN INFANTIL O PARVULARIA
}

\author{
Evaluation for learning in child or preschool education
}

\section{A avaliação para a aprendizagem em educação infantil ou pré-escola}

Rivera Machado Anais Yaned. Universidad del Tolima, Ibagué, Colombia. Fono: 3152456291. Correo electrónico: ayriveram@ut.edu.co

García Carrillo Luz Stella. Universidad del Tolima. Ibagué, Colombia. Fono: 3103490066. Correo electrónico: 1sgarcia@ut.edu.co

\section{Resumen}

Este artículo pretende presentar la incidencia e importancia de la evaluación como un proceso para el aprendizaje y el desarrollo de las competencias en los niños y niñas de la educación infantil o Parvularia orientadas por el educador al momento de diseñar y ejecutar la organización de su actividad pedagógica y didáctica. Para ello se realizó un estudio documental y reflexivo de diferentes experiencias de investigación presentadas por estudiantes en su práctica pedagógica en centros de atención en Ibagué, Colombia.

Se consideraron los planteamientos y referentes teóricos expuestos por algunos autores quienes presentan sus aportes frente al tema con un acercamiento conceptual a la evaluación del aprendizaje en la educación infantil o Parvularia.

Como reflexión final se puede afirmar que es importante integrar y articular coherentemente los elementos que componen el currículo por competencias, ya que en muchos casos se deja de lado la articulación de la educación infantil a los demás niveles educativos. Además es fundamental destacar la pertinencia de aplicar una evaluación formativa y sistemática de las estudiantes acordes al contexto, características, condiciones y necesidades

Palabras clave: evaluación para el aprendizaje; competencias; educación infantil; educación parvularia. 


\section{Summary}

This article pretends to show the incidence and importance of evaluation, as a process for learning and developing competences in child and preschool education orientated for the teacher at the moment of design and execute the organization of their pedagogical and didactic activity. In order to do it, a documentary and reflexive study of the different experiences of researching was developed, presented by students in their pedagogic internship centers in Ibagué, Colombia.

It was considered the theorical references presented by some authors who showed their knowledge about the subject, with a conceptual approach to evaluation of learning in child and preschool education.

As a final reflexion it can be affirmed that is important to integrate and articulate with coherence the elements that compose the curriculum by competences, because in many cases it is leave on a side the transition of children education, with higher educational levels. Furthermore is really important to exalt to students the pertinence to use a formative and systematical education according to the context, their characteristics, conditions and necessities.

Keywords: evaluation for learning; competences; child education.

\section{Resumo}

Este artigo tem como objetivo apresentar a incidência ea importância da avaliação como um processo de aprendizagem e desenvolvimento de habilidades em crianças de jardim de infância ou pré-escola educador direcionados ao projetar e implementar a organização de suas atividades de ensino e didática. Para fazer um documentário e pensativo estudo de experiências de pesquisa diferentes feitos por estudantes em sua prática de ensino em centros de cuidados em Ibague Colômbia foi feito.

Abordagens e referências técnicas expostas por alguns autorers que apresentam suas contribuições para o tema através de uma abordagem conceitual para a avaliação da aprendizagem na educação infantil e pré-escolar foram consideradas. 
Como um pensamento final pode dizer que é importante integrar e articular de forma consistente os elementos do currículo de competência, uma vez que em muitos casos é deixado de fora do pré-escolar conjunta para outros níveis de ensino. É também essencial para enfatizar a relevância da implementação de um contexto de avaliação formativa coerente e sistemática, as características, condições e necessidades.

Palavras-chave: avaliação de aprendizagem; compentencias; jardim de infancia; préescola.

\section{Introducción}

La educación infantil o parvularia se desarrolla en un panorama complejo, afronta diferentes retos y problemas al igual que los otros niveles como la Educación Básica y media. La labor profesional del docente, que si bien cuenta con una formación disciplinar y se observan avances significativos, aún persisten algunas limitaciones en su quehacer cotidiano y en las concepciones y prácticas de evaluación formativa.

Es incuestionable el interés que despierta y la preocupación del estado y de los entes internacionales por la educación infantil, reflejado en la promulgación de políticas educativas nacionales y de los organismos multinacionales que desde mediados del siglo pasado, discuten sobre su cobertura, calidad y pertinencia; aspectos que generan muchos interrogantes.

Otro aspecto a tener en cuenta es la diversidad de denominaciones y periodos de tiempo en que se ubica este nivel educativo, tales como educación infantil, educación inicial, educación para la primera infancia y educación Parvularia entre otros de acuerdo a la legislación educativa de cada país y las tendencias teóricas. Para el presente texto acopiamos el término educación infantil o Parvularia.

En muchas instituciones la labor de enseñanza aprendizaje se ha centrado en la realización de actividades manuales, de teorización y memorización mecánicos que generan la pérdida de la intencionalidad formativa en la educación infantil. Esta etapa es muy importante porque el ritmo de aprendizaje en este periodo es muy rápido e intenso, el infante aprende y experimenta un desarrollo acelerado de sus habilidades, competencias y destrezas 
específicamente cognitivas, sociales, comunicativas, científicas, motrices y ciudadanas y adquiere las competencias básicas para un acorde desarrollo en su vida futura.

Por ejemplo en Colombia, la renovación curricular y las exigencias del Ministerio de Educación Nacional destacan la importancia de desarrollar competencias específicas para el preescolar y transición como se plantea en los Lineamientos Curriculares, la Ley de Infancia y Adolescencia, y la reglamentación del programa interinstitucional De Cero a Siempre como política pública para la atención integral de la infancia.

Además, desde las universidades formadoras de profesionales en Educación, se evidencia el interés para realizar prácticas pedagógicas de observación y de intervención en instituciones donde se ofrece el servicio a niños y niñas en diferentes ambientes, por ello es necesario saber qué sucede en el desarrollo de las competencias en las instituciones y cuál es el sentido de la evaluación que allí se genera.

Así mismo es indispensable enfatizar que el docente y su quehacer en el ambiente escolar son fundamentales para el logro de los propósitos que propendan por el desarrollo y crecimiento de los niños lo que beneficiara el estudiante y también el docente. De ahí la importancia que los docentes apliquen estrategias de enseñanza y de evaluación formativa que incidan en la apropiación de las competencias. Si el docente está en permanente capacitación, formación y cualificación respecto a su quehacer participando en cursos, talleres y diplomados que le permitan fortalecer o mejorar su labor para lograr aprendizajes significativos y comprensivos desde una evaluación formativa.

Porque, en la medida que los docentes formen ciudadanos libres y responsables estaremos en posibilidad de mejorar las formas de vida, el desarrollo de competencias, y además, se puede aportar a la transformación y resolución de los problemas sociales, familiares y de aprendizaje que enfrentan nuestros niños y sus familias al iniciar esta etapa tan fundamental para su formación académica, personal y social.

\section{La Educación Infantil o Parvularia}

La Educación infantil constituye la primera etapa de la formación escolarizada o no del niño. Entre sus principios se considera el respeto a las necesidades e intereses de los niños. 
Tiene como finalidad contribuir en la adquisición y desarrollo de la capacidad de expresión para un aprendizaje reflexivo, un pensamiento crítico y creativo de los niños y niñas donde es decisivo y fundamental el juego, la fantasía y la percepción del mundo que le rodea para favorecer su proceso de socialización; los infantes están ansiosos de comprender lo que sucede, lo que les rodea, e identifica lo que el mundo les presenta día a día, se encuentran a la expectativa de hallar respuestas satisfagan en su mundo personal.

Teniendo en cuenta que en la educación infantil es indispensable ver al niño y niña de forma holística desde las dimensiones para el desarrollo humano, por tanto los niños desde que nacen, están en disposición de recibir y construir un nuevo conocimiento, y lo logran por sí mismos, pero en especial con la mediación de sus pares y adultos. Es necesario pensarlos como seres en construcción, con dudas, con ganas de explorar, con expectativas de formar parte de la sociedad del conocimiento y poder disponer del andamiaje necesario para dar pasos certeros en su desarrollo y formación.

Por ejemplo en el caso Colombia desde la mirada y planteamientos de los Lineamientos Curriculares del nivel preescolar (1997), se dice:

La educación preescolar deber animar, en forma permanente, la reflexión y el cuestionamiento de los docentes; detectar sus actitudes y posicionamiento frente a la cultura en que se integran con el fin de identificar prejuicios o tendencias hacia ella; provocar la búsqueda de valores y características esenciales de la cultura en donde viven los niños; realizar un proceso de integración, selección y filtro de lo más valioso y significativo de ella para el proceso educativo y analizar su compatibilidad con las de otras fuentes culturales; valorar otros agentes educativos, reconocer ambientes físicos propios de la comunidad, estructurar, tiempos y espacios acordes con sus significaciones en la comunidad.

\section{La evaluación como aprendizaje}

Es importante no perder de vista el reconocimiento que progresivamente gana la evaluación educativa en sus diferentes ámbitos, por ello, se requiere desarrollar iniciativas que permitan estudiar, debatir y reflexionar sobre el tema para resignificar y superar la mirada reduccionista e instrumental de la evaluación como simplemente realizar una serie de preguntas o exclusivamente asignar una calificación o nota. Por ello para no continuar en la zaga respecto a la evaluación educativa y en particular en la evaluación en la Educación 
Infantil o Parvularia es conveniente promover la llegada al aula de otros planteamientos teóricos y prácticas evaluativas.

Se impone entonces, avanzar en una remozada comprensión de la evaluación como aprendizaje y no como un enunciado respecto a cómo debe ser, sino ante todo como un ejercicio práctico para el aprendizaje, que se pone en marcha cotidianamente con un carácter eminentemente pedagógico, ético y político. Es decir, “debe ser un proceso que acompaña el aprendizaje, permite la construcción y refuerzo del conocimiento y una posibilidad de aprender del error. "No es un fenómeno ajeno, añadido, sobrepuesto y descontextualizado." (Santos Guerra, 2010, p. 12), no como un evento final y descontextualizado o una serie de actividades aisladas.

El Ministerio de Educación Nacional (2010) se define:

La evaluación es un proceso que permite la observación, identificación y seguimiento de un conjunto de competencias que los niños evidencian cuando se enfrentan a las actividades propuestas, además de rescatar lo que los niños ya poseen o conocen. Así mismo es una actividad importante dentro del proceso de enseñanza, porque contribuye a orientar el trabajo de las maestras en el aula (p.17)

Evaluar no es sinónimo de calificar aunque durante muchos años esto se ha hecho, se debe esclarecer que evaluar supone: por un lado calificar para dar un resultado expresado en una nota dado que la evaluación tiene como una de sus funciones reconocidas socialmente el calificar para certificar el aprendizaje. Por otro lado, y al mismo tiempo, simultáneamente pero con finalidades diferentes, evaluar supone un proceso de valoración, del proceso de formación. Es decir, calificar y evaluar son dos actividades complementarias.

La evaluación formativa cambia radicalmente su finalidad y desarrollo en la evaluación de los estudiantes, se prioriza la necesidad de ir más allá en el aprendizaje, acompañando y trabajando para posibilitarlo, en un intento para superar las posturas que solo evalúan el resultado, además, no es seguir un manual de procedimientos operativos para definir quién es promovido o no y solamente aplicar una prueba o un test.

La evaluación formativa como aprendizaje debe ser entendida como un proceso estructurado, planificado, situado, continuo que aporta al aprendizaje y permite obtener 
información para conocer la evolución del estudiante, sus avances y dificultades. Tiene como finalidad por un lado asignar una nota o calificación y por otro, valorar y favorecer el aprendizaje, corregir, retroalimentar al profesor y al estudiante para mejorar los procesos formativos. (García Carrillo, 2015)

Es entonces como la evaluación se convierte en un proceso dinámico inherente a la práctica educativa, que permite al educador valorar la tarea educativa y recibir información para tomar decisiones para mejorar su práctica docente. Como lo confirman, Velásquez y Hernández (2004) que insisten en que la evaluación como aprendizaje, se recomienda sea eminentemente formativa, ya que:

No posee un carácter sancionador con respecto a los resultados de aprendizaje, sino más bien un carácter indagador en relación con el propio proceso de enseñanza y aprendizaje, permite obtener información y disponer de conocimiento sobre lo que está sucediendo en un determinado momento del proceso y, en consecuencia, ayuda a la reflexión y a la toma de decisiones didácticas sobre la marcha (p.30)

Según Díaz y Hernández (2002) al referirse la evaluación de los aprendizajes se plantean dos tipos de funciones: la pedagógica y la social. En los grados de preescolar y básica primaria su función es eminentemente pedagógica para obtener la información necesaria para valorar el proceso educativo, la práctica pedagógica y los aprendizajes de los estudiantes con la finalidad de tomar decisiones sobre las acciones y realizar las mejoras pertinentes.

La evaluación se convierte en un proceso continuo, permanente y dialogado, que "permite generar estrategias educativas que ayudan a los niños a progresar y a transformar las dificultades que se les presentan” (Ministerio de Educación Nacional, 2010, p.17).

De la misma manera como se evalúan los actores es importante destacar la evaluación del proceso educativo y formativo en la Institución educativa, es decir, las acciones realizadas en el nivel inicial en la cual se toma como referencia la evaluación de los aprendizajes de los estudiantes con la finalidad de dirigir las acciones del directivo y docentes para el logro de mejores resultados e invitar a una reflexión colegiada y dialogada atendiendo a los 
principios de la evaluación en la práctica docente y en el trabajo en el aula y en la escuela que permiten ver si es adecuada la organización y funcionamiento de la misma.

Además, conviene dar una mirada a la evaluación diagnóstica que es básica en la educación infantil, por tanto el docente debe centrar su atención en identificar el nivel de competencias que muestran los estudiantes al iniciar el programa, y de esta manera elaborar la planificación del proceso la enseñanza aprendizaje orientado a las necesidades y dificultades de cada uno de los infantes en su contexto y para seleccionar las estrategias de intervención pedagógica y didáctica más pertinentes.

A sí mismo el principal protagonista en la evaluación es el educador ya que a partir del conocimiento que tiene de sus estudiantes, diseña, planea, coordina, orienta y da seguimiento a las acciones y actividades de apoyo pertinentes a realizar en el aula para el logro de las competencias y desempeños. La evaluación diagnóstica se debe llevar a cabo durante el primer mes de trabajo con los niños para lo cual el docente de la educación infantil diseña un plan mensual con actividades exploratorias que abarcan competencias de los diferentes campos formativos para poder determinar el nivel de dominio que tienen los estudiantes.

Así mismo es importante tener en cuenta las opiniones de los niños, acerca de las dificultades presentadas en el desarrollo de las actividades, las sugerencias o gustos por ciertas actividades. Los padres de familia también aportan elementos valiosos sobre los avances y retrocesos que detectan en sus hijos, sobre las acciones que emprende el docente, son comentarios que permiten revisar las formas del funcionamiento y organización curricular y el trabajo realizado en el aula.

En el mismo sentido la evaluación formativa como aprendizaje por su carácter cualitativo utiliza principalmente la técnica de la observación directa se recomienda que el educador realice registros sobre los aspectos más relevantes para construir la historia personal de su estudiante, es un proceso de obtener, sintetizar e interpretar información para facilitar la toma de decisiones orientadas a ofrecer retroalimentación al estudiante, es decir, para mejorar el aprendizaje durante los diferentes periodos de enseñanza. 
En la educación infantil, la función de la evaluación es eminentemente didáctica y pedagógica ya que se realiza para obtener la información necesaria para valorar el proceso formativo, la práctica pedagógica y los aprendizajes de los estudiantes, y debe:

- Constatar los aprendizajes de los estudiantes, sus aciertos y dificultades que manifiestan para alcanzar las competencias básicas en función del ritmo y estilo de aprendizaje que esta intermediado por el contexto.

- Identificar los factores que influyen o afectan el aprendizaje de los niños y niñas, las formas de evaluar que desarrollan, las condiciones del ambiente familiar y social como base para valorar su pertinencia o su modificación. Por tanto, es esencial acercarse a los diferentes contextos para conocer de cerca la realidad imperante en los contextos de la educación infantil. Al respecto Álvarez Méndez (2012)

"Se trata, en la nueva lectura y en una interpretación ingenua en clave positiva, de evaluar para aprender, evaluar para enseñar a aprender y para enseñar a estudiar de tal modo que puedan evitarse errores de procedimiento en el proceso de aprender, evaluar para facilitar y asegurar el aprendizaje"

En este sentido las estrategias y apoyos metodológicos para la evaluación en la educación infantil, son indispensables en el proceso de formación. Escudero (1997) indica en una de sus propuestas que es primordial dedicarse a los productos, a ver los antecedentes y el proceso. Este enfoque más formativo y más holístico permiten la mejora real del sistema. Implica fusionar la evaluación diagnóstica, la formativa y la sumativa, implementando tanto la dimensión personal y académica, teniendo en cuenta que los niños y niñas se encuentran en una etapa de transición entre el mundo familiar y los primeros años de escolaridad.

Ahora bien, al colocar la evaluación al servicio del aprendizaje, se debe tener en cuenta que "el aprendizaje, consecuente y simultáneamente, la evaluación, deben estar orientados y dirigidos por el currículo...y por la enseñanza que debe inspirarse en él”. (Álvarez, 2001, p.34)

Desde esta mirada, Miguel Ángel Santos Guerra plantea “convertir la evaluación en un proceso permanente de revisión y análisis de la práctica. Porque la evaluación es una fuente 
en si misma de interrogantes pero, además, contiene en su dinámica elementos suficientes para poner en cuestión toda la concepción curricular” (Santos, 2010, p. 42)

\section{Para concluir}

Para concluir es fundamental insistir en la pertinencia de aplicar una evaluación formativa como aprendizaje de forma sistemática a los estudiantes en la Educación Infantil acorde al contexto, las características, las condiciones y las necesidades.

Así mismo es necesario trabajar por integrar y articular coherentemente la Educación Infantil a los elementos que componen el currículo institucional, ya que en muchos casos se deja de lado la articulación entre los niveles de la Educación Infantil con los demás grados. Allí la evaluación de los aprendizajes debe ser tenida en cuenta en el sistema de evaluación

y promoción de los estudiantes, la evaluación debe ser planeada para integrarla y desarrollarla de manera flexible, comprensiva y transversal pero dejando claro que en este nivel todos los niños deben ser promovidos.

Pero, ante todo es imprescindible invertir en la formación inicial y continuada en evaluación de los docentes y el personal encargado de desarrollar los diferentes procesos formativos en la Educación Infantil, este es un factor determinante en la transformación del significado y sentido de la evaluación de los estudiantes, además, de la promoción y apoyo de investigaciones que aporten a tal fin.

\section{Referencias bibliográficas}

Álvarez J. M. (2001) Evaluar para conocer, examinar para excluir. Madrid: Morata.

Álvarez J. M. (2009). La evaluación en la práctica de aula. Estudio de Campo. Revista de Educación. 350. Septiembre - diciembre 2009.

Coll, C. (2007) Las competencias en la educación escolar: algo más que una moda y mucho menos que un remedio. Revista Aula de Innovación Educativa, núm. 161. España.

Escudero T. (1995) Evaluación de los aprendizajes en la Universidad. En Actas del Symposium de Innovación Universitaria 
Díaz Barriga F. y Hernández Rojas, G. (2002) Estrategias docentes para un aprendizaje significativo. Una interpretación constructivista. México: McGraw-Hill

García Carrillo L. S. (2015) La evaluación formativa una experiencia para el aprendizaje en la Educación Física escolar. Taller en Expomotricidad. $6^{\circ}$ Simposio Internacional de Educación Física. Itinerarios que dotan de sentido el hacer en el aula. Medellín.

(Compiladora) (2012) La Evaluación escolar. Una práctica cotidiana que va perdiendo el año. Ibagué:Editorial Universidad del Tolima.

(2012) Principios para una evaluación formativa en la Educación Física escolar. Revista Kinesis: Ciencias del deporte, Educación Física y Recreación. Número 59: Armenia.

Ministerio de Educación Nacional (2010) Aprender y jugar, Instrumento Diagnostico de Competencias Básicas en Transición. Documento N 13.Bogota.

Ministerio de Educación Nacional (1997) Lineamientos Curriculares para el preescolar.

Serie Lineamientos Curriculares. Bogotá.

Moreno T. (2011) Frankestein evaluador. En Revista de la Educación Superior. Vol. 40, núm. 160. México, octubre - diciembre/2011.

Perrenoud, P. (2007) Diez nuevas competencias para enseñar (4ª ed.). Barcelona: Graó.

Rivera A. Y. (2015) La evaluación dinamizadora de las competencias comunicativas en la transición de la Básica a la universidad. Ponencia I Coloquio Internacional de Educación a Distancia y Educación Virtual. Ibagué.

Velásquez R. y Hernández J. L. (2004) La evaluación en Educación Física. Investigación y práctica en el ámbito escolar. Barcelona: Grao.

Santos Guerra M. A. (2010) La evaluación como aprendizaje. Una flecha en la diana. Tercera edición. Bonum. 
Infancia, Educación y Aprendizaje (IEYA). Vol. 2, No 2, pp. 106-117. ISSN: 0719-6202 http://revistas.uv.cl/index.php/IEYA/index 